\title{
A system for remote dosimetry audit of 3D- CRT, IMRT and VMAT based on lithium formate dosimetry
}

Emelie Adolfsson, Håkan Gustafsson, Eva Lund, Gudrun Alm Carlsson, Sara Olsson and Åsa Carlsson Tedgren

\section{Linköping University Post Print}

\section{Tweet}

N.B.: When citing this work, cite the original article.

Original Publication:

Emelie Adolfsson, Håkan Gustafsson, Eva Lund, Gudrun Alm Carlsson, Sara Olsson and Åsa Carlsson Tedgren, A system for remote dosimetry audit of 3D-CRT, IMRT and VMAT based on lithium formate dosimetry, 2014, Radiotherapy and Oncology, (113), 2, 279-282.

http://dx.doi.org/10.1016/j.radonc.2014.11.027

Copyright: Elsevier http://www.elsevier.com/

Postprint available at: Linköping University Electronic Press http://urn.kb.se/resolve?urn=urn:nbn:se:liu:diva-111089 


\title{
A system for remote dosimetry audit of 3D-CRT, IMRT and VMAT based on lithium formate dosimetry
}

\author{
Emelie Adolfsson ${ }^{1}$, Håkan Gustafsson ${ }^{2}$, Eva Lund ${ }^{3}$, Gudrun Alm Carlsson ${ }^{3}$, Sara Olsson ${ }^{4}$, Åsa \\ Carlsson Tedgren ${ }^{5}$
}

${ }^{I}$ Department of Radiation Physics and Radiation Physics, Department of Medical and Health Sciences, Linköping University, Linköping, Sweden

${ }^{2}$ Department of Medical Technology (MTÖ), Radiation Physics, Department of Medical and Health Sciences, Linköping University, Linköping, Sweden

${ }^{3}$ Radiation Physics, Department of Medical and Health Sciences, Center for Medical Image Science and Visualization, Linköping University, Linköping, Sweden

${ }^{4}$ Medical Physics and Technology, Växjö Central Hospital, Växjö, Sweden

${ }^{5}$ Swedish Radiation Safety Authority, Stockholm, Sweden, and Radiation Physics, Department of Medical and Health Sciences, Linköping University, Linköping, Sweden

$\begin{array}{ll}\text { Corresponding author: } & \text { Emelie Adolfsson } \\ \text { Telephone } & +46101031793 \\ \text { E-mail } & \text { emelie.adolfsson@liu.se } \\ \text { Mailing address } & \text { Radiation Physics, O-huset Plan 8 } \\ & \text { SE 581 85 Linköping, Sweden }\end{array}$

Key-words: Dosimetry audit; remote audit; end-to-end; EPR; ESR; lithium formate

Number of tables: 0

Number of figures: 3

Running head: A system for remote dosimetry audit

\section{Summary}

The aim of this work was to develop and test a remote end-to-end audit system using lithium formate EPR dosimeters. Four clinics were included in a pilot study, absorbed doses determined in the PTV agreed with TPS calculated doses within $\pm 5 \%$ for $3 \mathrm{D}$-CRT and $\pm 7 \%(\mathrm{k}=1)$ for IMRT/VMAT dose plans.

\section{Introduction}

The introduction of advanced treatment techniques such as intensity modulated radiotherapy (IMRT) and volumetric modulated arc therapy (VMAT) has introduced new challenges for dosimetry audits and also increased the importance of auditing. The two largest audit networks are currently operated by the IAEA [1, 2] and the Imaging and Radiation Oncology Core Huston (IROC Huston) [3].

Electron paramagnetic resonance (EPR) dosimetry using alanine is an accepted standard dosimetric method and has been used for audits in the UK [4], Italy [5] and Belgium [6]. The readout process for EPR dosimeters is non-destructive to the signal which allows several readouts of the same dosimeter. Lithium formate is thoroughly investigated and has dosimetric properties at least as good as those of alanine. Both lithium formate and alanine has a linear dose response in a large dose region $(0.2 \mathrm{~Gy}$ 1000 Gy). This enables measurements of both high and low doses simultaneously. Most audit systems 
found in the literature focus on dose determination in absolute terms solely at the isocentre. However, the doses to organs at risk (OARs) are equally important to verify, since they normally set the limit for the target dose. Compared to alanine, lithium formate has an even better water equivalence regarding mass-energy absorption and mass collision stopping power and also up to seven times higher sensitivity $[7,8]$. The lithium formate system is robust; the signal is stable at least one month after irradiation (a sufficient time period for audit measurements) and not affected by variations in storage temperature [9]. The response is independent of dose rate and beam quality in the MV energy region [10]. Lithium formate has previously been used for verification of IMRT treatments [10] and for measurement around ${ }^{192} \mathrm{Ir}$ brachytherapy sources [11]. The LET-dependence of response for photon energies $<150 \mathrm{keV}$ relative to ${ }^{60} \mathrm{Co}$ is known [12] and imply that the lithium formate system is a good candidate for use also in brachytherapy audits.

The aim of this work was to develop and evaluate a remote end-to-end audit system for high energy external beam therapy using lithium formate EPR dosimeters with determination of doses in absolute terms in target and OARs for 3D-CRT, IMRT and VMAT. The system was tested in a pilot study at four clinics in Sweden. A future aim is to participate in developing a system for quality improvements of radiotherapy that can serve useful in fulfilling upcoming national regulatory requirements on regular, independent dosimetric audits.

\section{Material and methods}

\section{Dosimeters and EPR readout}

The cylindrical dosimeters pressed to a height of $4.8 \mathrm{~mm}$ and diameter $4.5 \mathrm{~mm}$ consist of $90 \%$ lithium formate and $10 \%$ paraffin used as a binder. A BRUKER EleXsys E500 was used with a BRUKER standard resonator (4102ST) for all readouts. Details about the dosimeter manufacturing and handling can be found in earlier publications [10-12].

\section{Phantom}

A semi-anthropomorphic head-and-neck phantom was designed with inspiration from Han et al. [13], figure 1 . The phantom included a tumour structure partially encompassing the spinal column (made of Teflon), two structures resembling salivary glands, and a small air cavity symbolizing the trachea. All structures except the Teflon spinal column were made of PMMA. The phantom was constructed of eight circular PMMA disks stacked together as a cylinder with diameter $20 \mathrm{~cm}$, length $24 \mathrm{~cm}$. The total phantom weight is $10 \mathrm{~kg}$. The structures are contained in the four central slabs (divided equally among them) and constitute the irradiated volume. The dosimeters were positioned in PMMA rods, which in turn were positioned at the six measurement points, three in the target volume, one in each salivary gland and one in medulla. Three dosimeters were used at each measurement point, positioned in a row in the cranial-caudal direction. 


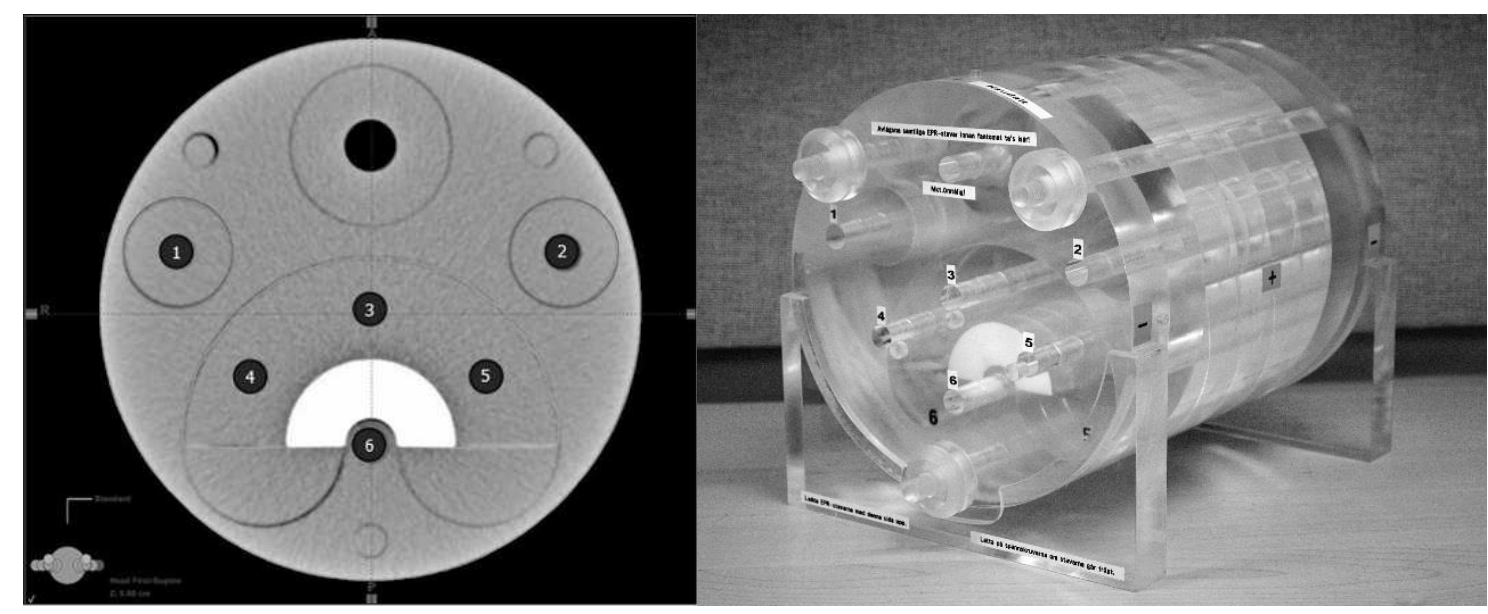

Figure 1. The audit phantom. The numbers in the CT slice indicate the measurement positions: salivary glands $(1,2)$, target $(3,4$, and 5$)$ and medulla $(6)$.

\section{Audit procedure}

Four clinics were included in the pilot study testing the audit procedure and the dosimetry system. A 3D-CRT audit was performed at all four clinics, IMRT at two clinics and VMAT at one. At the time of the study, IMRT and VMAT had not been implemented at the other clinics.

The dosimeters were prepared and loaded into their PMMA rods. The phantom, dosimeters and instructions were sent by mail to the audit sites. The phantom went through CT-scanning, contouring, dose planning and treatment delivery. During the CT-scan, solid PMMA rods were inserted in the phantom to avoid the extra dose from the CT. (Tests have shown that no difference in the HU-value was detected between lithium formate and PMMA at the particular positions). The structures appear clearly on the CT scans and the contouring should not affect the outcome of the audit. The dose planning in this pilot test was restricted to include a certain number of fields, energies, wedges etc. The planned dose to the PTV was set to 2 Gy to be delivered twice, a 4 Gy total dose to achieve high EPR signal to noise ratio. The standard 2 Gy per fraction was chosen to keep the multi-leaf collimator (MLC) speed clinically relevant. The institutions were asked to report the planned doses together with the daily output of the particular beam used for irradiation.

\section{Uncertainty budget and acceptance criteria}

Absorbed dose to water determined by the dosimeters has a combined standard uncertainty of $2 \%-2.5 \%$. The main contributing factor is the type B uncertainty of the ionization chamber used for calibration of the dosimeters $(1.7 \%)$ [14], other factors are related to the readout process and the homogeneity within the dosimeter batch. According to a review by Ahnesjö and Aspradakis [15], the relative standard uncertainty in the treatment planning system (TPS) dose calculation is approximately $2 \%(\mathrm{k}=1)$. Thus, the relative combined standard uncertainty of the percentage deviation between measured and calculated doses is $3 \%(\mathrm{k}=1)$ [16]. This uncertainty level was considered sufficient to set a preliminary 
acceptance criterion of 5\% for 3D-CRT. The dose calculation, planning and delivery of IMRT and VMAT are more complex compared to 3D-CRT and are associated with larger uncertainty [17]. Thus, the preliminary acceptance was set to $7 \%$ for IMRT and VMAT.

\section{Results and discussion}

Results from the pilot study are shown in figures 2 (3D-CRT) and 3 (IMRT, VMAT).

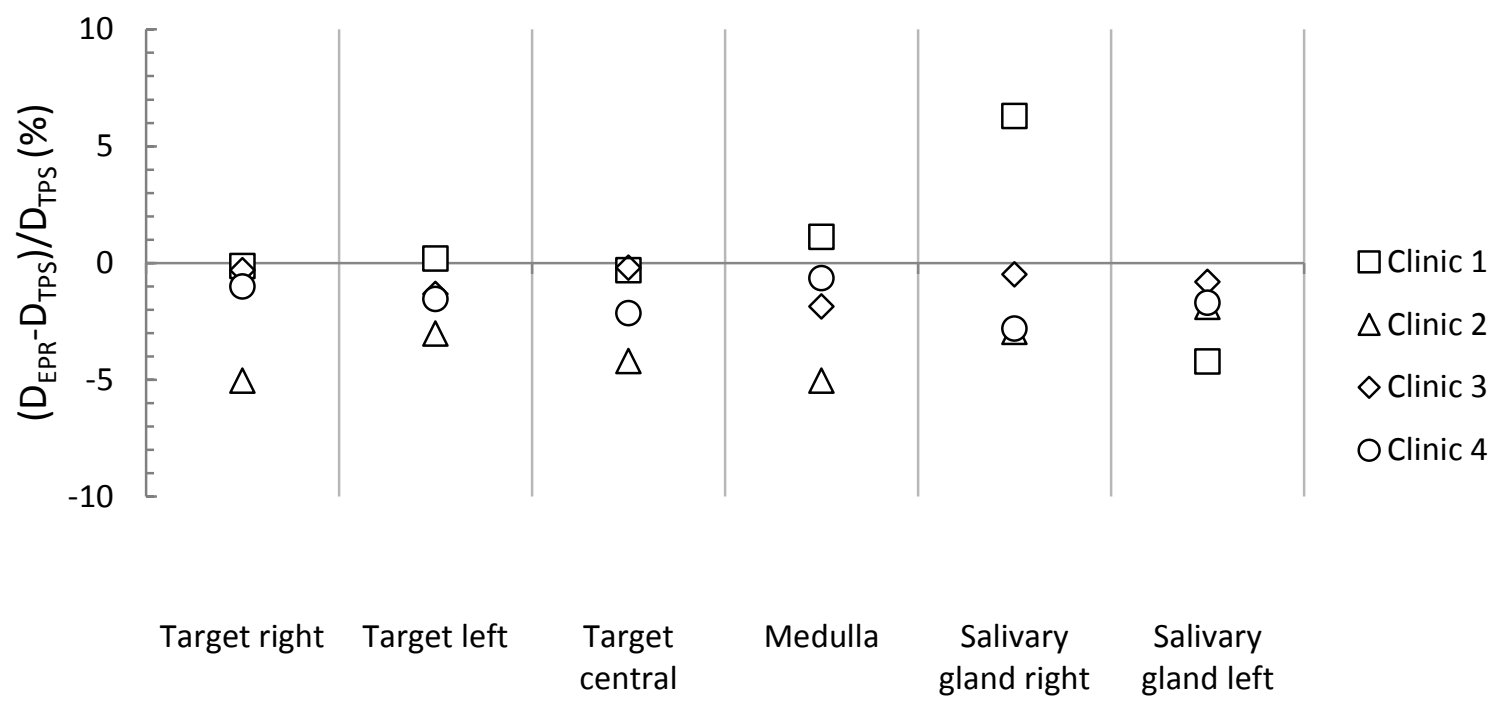

Figure 2. 3D-CRT: Percentage difference between measured (EPR) and calculated (TPS) absorbed doses in each measurement point.

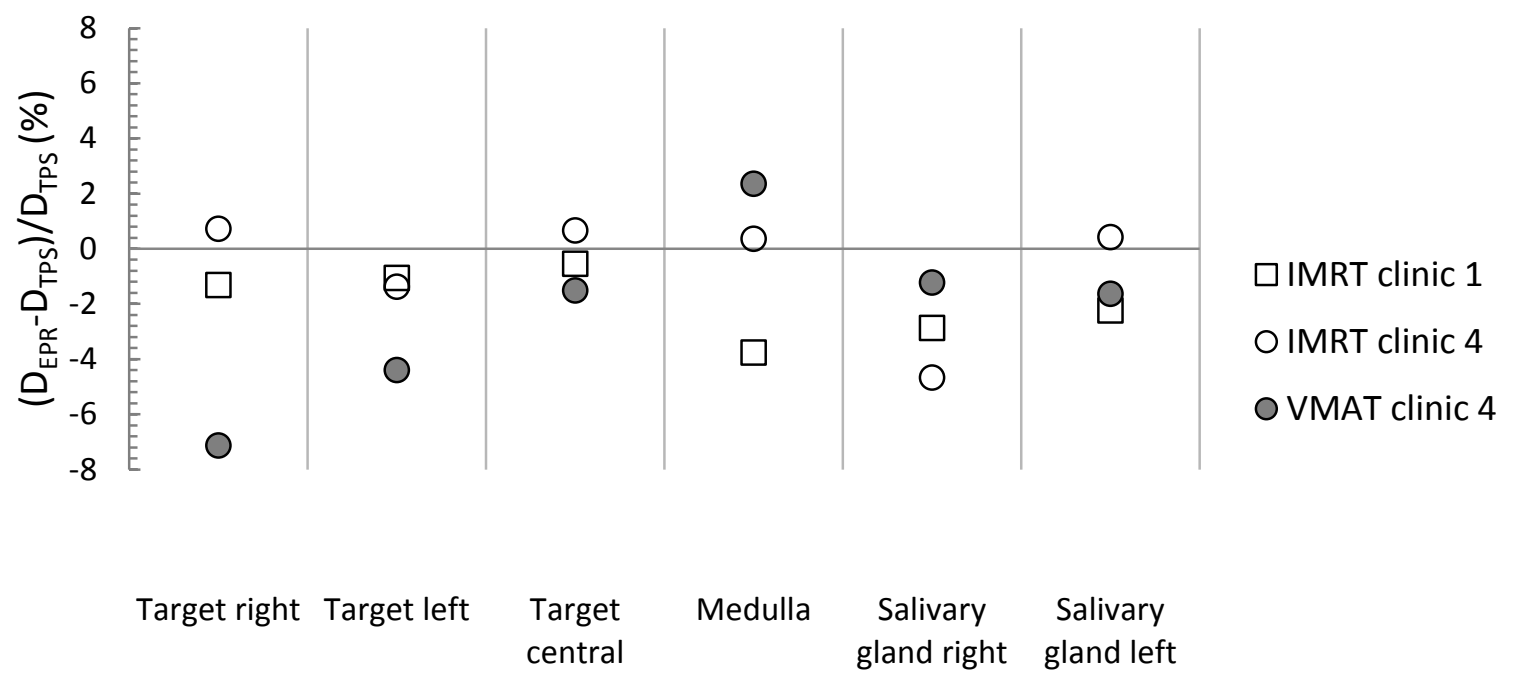

Figure 3. IMRT and VMAT: Percentage difference between measured (EPR) and calculated (TPS) absorbed doses in each measurement point.

The absorbed doses at all measurement points are within the $\pm 5 \%$ criterion for the 3D-CRT measurements for clinics 3 and 4. For the IMRT/VMAT results, all measurement points were within 
$\pm 7 \%$ but some close to the acceptance limit. This irradiation should preferably be repeated to determine the reproducibility in the result before any conclusions can be drawn. It could be discussed whether one should have acceptance criteria for both PTV and OARs. IROC Huston has 7\% as acceptance for measurement points in PTV (end-to-end IMRT, head and neck phantom), but only gamma criterion for measurement points in the OAR $[18,19]$. Even though it is important to verify the dose to OARs, it is normally more difficult to measure doses with high accuracy due to the dose gradients that normally appear in them. Also, small deviations in absolute dose can be large in percent. An alternative could be to set a percentage of the PTV dose for acceptance or to have higher acceptance limits for the OARs.

Clinic number 1 used the Elekta Oncentra (Elekta AB, Stockholm, Sweden) TPS with the collapsed cone (CC) algorithm and dose were delivered using Varian $2100 \mathrm{C} / \mathrm{D}$ linear accelerator. Clinics number 2, 3 and 4 used the Varian Eclipse (Varian Medical Systems, Palo Alto, California, USA) TPS with the AAA algorithm and Varian accelerators 600 C/D (clinic 2), 23EX (clinic 3) and iX (clinic 4). It can be noted that although all deviations are within the acceptance criteria, all measurements except those at clinic 1 are showing results below unity, i.e. measured doses are lower than calculated ones. Following findings by Fransson and Johnsson [20] on how the CT calibration curve of the TPS affects AAA calculated doses, we recalculated the plans from clinic 2 and 4, using a CT calibration curve obtained with acrylic calibration inserts instead of the Varian AAA default curve aimed for patient calculations. This resulted in reduced calculated doses of approximately $2 \%$ for 3D-CRT and $1.5 \%$ for IMRT and VMAT plans (varying with depth in the phantom) and hence in improved agreement between measured and calculated doses. Thus, part of the deviations between measured and AAA calculated doses seem to stem from the use of a PMMA phantom together with the Varian default CT calibration curve. In an end-to-end dosimetric audit, it is essential that the patient CT calibration curve is used in TPS dose calculation as this is one of the factors desirable to control. The current finding suggests that use of a different plastic, closer to tissue in atomic composition than PMMA, would be a better choice.

One of the 3D-CRT audits had to be repeated twice before it was discovered that the out-of-tolerance results was a result of inclusion of couch rails in two of the radiation fields. This resulted in an underdosage of $12 \%$ in the salivary gland structures that was situated in the direct beam of these fields. This is an error that was discovered by the audit and is an error that can be present for patients as well. It should be mentioned that the audit irradiations were performed by physicists that do not normally treat patients.

In this pilot test, the dose planning process was chosen to follow certain criteria to be able to compare the results. For future measurements, the clinics should use their own treatment protocols. 


\section{Conclusions}

The developed audit system based on lithium formate EPR and the logistics around it were found suitable for end-to-end audits. Dose values measured in the audit agreed with TPS calculated doses in the PTV to within $\pm 5 \%$ for $3 \mathrm{D}$ CRT and $\pm 7 \%$ for IMRT/VMAT for all participating clinics. Part of the found deviations between doses calculated by the AAA algorithm and measured ones most likely stems from the use of a PMMA phantom together with the clinically used CT calibration curve. A phantom made of a plastic more close to the composition of tissue than PMMA might thus be preferred in future audits. Our audit system provides dose verification at six measurement points in absolute dose units. As a complement, the system could be extended to include gamma-analysis and dose profiles in $2 \mathrm{D}$ or $3 \mathrm{D}$, using radiochromic film.

\section{Acknowledgements}

This work was supported by grants from the Swedish Cancer foundation (CF), contract number 110322 and FORSS contract numbers 234291 and $86231 \mathrm{H}$. Gustafsson acknowledges The Swedish Research Council (diarienr 2009-5430). The authors thank Bengt Frost (County Council of Östergötland) for constructing the phantom and Zelga Malke, Mattias Karlsson and Rebecca Edén for valuable measurement contributions.

\section{References}

1. Izewska J, Andreo P, Vatnitsky S and Shrott KR. The IAEA/WHO TLD postal dose quality audits for radiotherapy: a perspective of dosimetry practices at hospitals in developing countries. Radiotherapy and Oncology 2003;69:91-97.

2. Izewska J, Bera P and Vatnitsky S. IAEA/WHO TLD Postal dose audit service and high precision measurements for radiotherapy level dosimetry. Radiation Protection Dosimetry 2002;101:387-392.

3. Ibbott GS. QA in Radiation Therapy: The RPC perspective. Journal of Physics: Conference Series 2010;250:012001.

4. Budgell G, Berresford J, Trainer M, Bradshaw E, Sharpe P and Williams P. A national dosimetric audit of IMRT. Radiotherapy and Oncology 2011;99:246-252.

5. De Angelis C, De Coste V, Fattibene P, Onori S and Petetti E. Use of alanine for dosimetry intercomparisons among Italian radiotherapy centers. Appl. Radiat. Isot. 2005;62:261-265.

6. Schaeken B, Cuypers R, Lelie S, et al. Implementation of alanine/EPR as transfer dosimetry system in a radiotherapy audit programme in Belgium. Radiotherapy and Oncology 2011;99:94-96.

7. Lund E, Gustafsson H, Danilzuk M, et al. Formates and dithionates: sensitive EPR-dosimeter materials for radiation therapy. Appl. Radiat. Isot. 2005;62:317-324.

8. Vestad TA, Malinen E, Lund A, Hole EO and Sagstuen E. EPR dosimetric properties of formates. Appl. Radiat. Isot. 2003;59:181-188.

9. Adolfsson E, Karlsson M, Alm Carlsson $\mathrm{G}$, et al. Investigation of signal fading in lithium formate EPR dosimeters using a new sensitive method. Phys. Med. Biol. 2012;57:2209-2217.

10. Gustafsson H, Lund E and Olsson S. Lithium Formate EPR Dosimetry for verification of calculated dose distributions prior to intensity modulated radiation therapy. Phys. Med. Biol. 2008;53:4667-4682. 
11. Antonovic L, Gustafsson H, Alm Carlsson G and Carlsson Tedgren Å. Evaluation of a lithium formate EPR dosimetry system for dose measurements around ${ }^{192}$ Ir brachytherapy sources. Med. Phys. 2009;36:2236-2247.

12. Adolfsson E, Alm Carlsson G, Grindborg J-E, Gustafsson H, Lund E and Carlsson Tedgren Å. Response of lithium formate EPR dosimeters at photon energies relevant to the dosimetry of brachytherapy. Medical Physics 2010;37 (9):4946-4959.

13. Han Y, Shin EH, Lim C, et al. Dosimetry in an IMRT phantom designed for a remote monitoring program. Medical Physics 2008;35:2519-2527.

14. Andreo P, Burns DT, Hohlfeld K, et al., Absorbed Dose Determination in External Beam Radiotherapy, IAEA TRS 398: An International Code of Practice for Dosimetry Based on Standards of Absorbed Dose to Water, in IAEA Technical Report Series no 398 (Vienna: International Atomic Energy Agency). 2000, IAEA: Vienna, Austria.

15. Ahnesjö A and Aspradakis M. Review: Dose calculations for external photon beams in radiotherapy. Phys. Med. Biol. 1999;44:R99-R155.

16. International Organization for Standardization I, Guide to the expression of uncertainites in measurements. 1995.

17. Cedric $\mathrm{XY}$ and Grace T. Intensity-modulated arc therapy: principles, technologies and clinical implementation. Physics in Medicine and Biology 2011;56:R31-R54.

18. Molineu A, Hernandez N, Nguyen T, Ibbott $G$ and Followill D. Credentialing results from IMRT irradiations of an anthropomorphic head and neck phantom. Medical Physics 2013;40:1-8.

19. Weber D, Vallet V, Molineu A, et al. IMRT credentialing for prospective trials using institutional virtual phantoms: results of a joint European Organization for the Research and Treatment of Cancer and Radiological Physics Center project. Radiation Oncology 2014;9:123.

20. Fransson K and Johnsson S. PD-0079 Treatment planning on phantoms and patients requires different CT-calibration curves. Radiotherapy and Oncology 2012;103, Supplement 1:S31. 